\title{
To Compare the Effects of Adjuvant and Neoadjuvant Chemotherapy on Outcome of Stage III Carcinoma Breast
}

\author{
Anupam Surya Anand1, Raju Kamlakarrao Shinde²
}

\author{
${ }^{1}$ Department of General Surgery, Jawaharlal Nehru Medical College, DMIMS, Wardha, Maharashtra, India. \\ ${ }^{2}$ Department of General Surgery, Jawaharlal Nehru Medical College, DMIMS, Wardha, Maharashtra, India.
}

\section{ABSTRACT}

\section{BACKGROUND}

At present, the ideal treatment of patients diagnosed to have carcinoma breast includes multimodal treatment therapy. However, the sequence of various modalities in the treatment of breast cancer varies according to the stage of the tumour at the time of presentation. Early breast cancer cases first undergo surgical treatment modality before systemic therapies, while advanced cases should undergo systemic therapies first followed by surgical interventions if possible. However, treatment of stage IIIA and IIIB patients (locally advanced disease) but having an operable lump poses a dilemma of whether to go for surgery first or systemic therapy first. We wanted to compare the outcome in terms of metastasis/recurrence between adjuvant and neo-adjuvant chemotherapy in selected cases of stage IIIA and stage IIIB carcinoma breast for a follow-up period of 1 year at a tertiary care hospital in central India.

\section{METHODS}

This is a comparative observational study conducted at Acharya Vinoba Bhave Rural Hospital (AVBRH) of Jawaharlal Nehru Medical College from October 2017 to Sept 2019, which included patients of TNM stage IIIA and IIIB breast carcinoma, half of whom were treated with adjuvant chemotherapy and other half treated with neoadjuvant chemotherapy along with standard surgical procedure like MRM/toilet mastectomy.

\section{RESULTS}

Distribution of patients according to presence of lymphovascular Invasion was done which was statistically non-significant. However, when disease recurrence or disease metastasis/mortality was compared with lymphovascular invasion in neoadjuvant group, it was found to be statistically significant ( $p$ value $=0.022$ ) In the adjuvant group $90 \%$ of patients belonged to stage IIIA while in neo-adjuvant group only $50 \%$ patients belonged to stage IIIA. Rest patients in both group belonged to stage IIIB. This difference in adjuvant and neo-adjuvant group was statistically significant. $(\mathrm{p}=0.022)$ In comparison of outcome in both adjuvant and neo-adjuvant chemotherapy, $5 \%$ patients of adjuvant group developed metastasis and died succumbing to it while another $5 \%$ developed recurrence during follow up. In the neo-adjuvant group 35\% patients developed distant metastasis or died due to disease while another $5 \%$ patient developed local recurrence in axilla for the disease. This difference in the outcome of two groups was statistically significant with $\mathrm{p}$ value of 0.013 .

\section{CONCLUSIONS}

In our study we found that for a locally advanced breast cancer patient (stage IIIA \&B) with an operable breast lump, adjuvant chemotherapy is superior than neoadjuvant chemotherapy with a significant $p$ value of 0.013 . Superior in terms of lesser distant metastasis/recurrence when we followed up the patient for 1 year after the completion of treatment.

\section{KEY WORDS}

Carcinoma Breast, Stage III, Locally Advanced Breast Cancer, Neo-Adjuvant, Adjuvant, Recurrence, Mortality, Chemotherapy, Anterior Chemotherapy
Corresponding Author: Dr. Anupam Surya Anand, Room No. 11, Raghobaji Boys Hostel, Sawangi, Wardha-442001, Maharashtra, India.

E-mail: anupam6411@gmail.com

DOI: $10.14260 /$ jemds/2020/112

Financial or Other Competing Interests: None.

How to Cite This Article:

Anand AS, Shinde RK. To compare the effects of adjuvant and neoadjuvant chemotherapy on outcome of stage III carcinoma breast. J. Evolution Med. Dent. Sci.2020;9(08):496-501, DOI: $10.14260 /$ jemds $/ 2020 / 112$

Submission 25-11-2019,

Peer Review 30-01-2020

Acceptance 05-02-2020,

Published 24-02-2020. 


\section{BACKGROUND}

Breast cancer has become most common female cancer worldwide contributing nearly a quarter $(25 \%)$ of all cancer cases, with an estimated 1.67 million new cancer cases diagnosed in 2012. Less developed countries have a little more number of breast cancer cases (883000 cases) compared to more developed countries (794 000). ${ }^{1}$ In Indian subcontinent too there is a significant increase in the incidence and associated morbidity and mortality as reported in global and Indian studies.2,3,4,5 Locally advanced breast cancer remains a clinical challenge as the majority of patients with this diagnosis develop distant metastases despite appropriate therapy. Patients with locally advanced disease encompass a wide range of clinical scenarios including advanced primary tumours (stage T4), advanced nodal disease (fixed axillary nodes or involvement of ipsilateral supraclavicular, infraclavicular, or internal mammary nodes), and inflammatory carcinomas. The prognoses of women with locally advanced breast tumours are also heterogeneous and depend on tumour size, extent of lymph node involvement.6,7

At present, the ideal treatment of patients diagnosed to have carcinoma breast includes multimodal treatment therapy. Chemotherapy can be given adjuvant or neoadjuvant. In India a major chunk of patients of breast cancer presents with advanced disease.8,9 Treatment guidelines of locally advanced disease varies in different guidelines. NCCN advocated all locally advanced cases to undergo anterior chemotherapy first then surgical therapy even if skin closure and surgery is feasible at the time of presentation $^{10}$. Whereas, ICMR guidelines advocates first surgical therapy in locally advanced cases provided skin closure is feasible and the tumour is operable and then chemotherapy adjuvantly. ${ }^{11}$ It is mainly that we are comparing outcome in terms of metastasis/recurrence/mortality between adjuvant $(n=20)$ and neo-adjuvant chemotherapy $(\mathrm{n}=20)$ in selected cases (Who fit in our inclusion and exclusion criteria) of stage IIIA and stage IIIB carcinoma breast for a follow up period of 1 year.

We wanted to study the outcome, assess the local recurrence, determine systemic metastasis and compare the outcome of chemotherapy as well as response in terms of recurrence and metastasis, of adjuvant and neoadjuvant chemotherapy of stage III carcinoma breast.

\section{METHODS}

This is a comparative observational study conducted at Acharya Vinoba Bhave Rural Hospital (AVBRH) of Jawaharlal Nehru Medical College from October 2017 to Sept 2019, which included patients of TNM stage IIIA and IIIB breast carcinoma, half of whom were treated with adjuvant chemotherapy and other half were treated with neoadjuvant chemotherapy along with standard surgical procedure like $\mathrm{MRM}$ /toilet mastectomy. Thereafter parameters required for evaluation of outcome were studied. Data was collected through preformed proformas. Patients aged less than 70 years with clinically palpable, primary breast cancer confirmed by Tru-Cut biopsy/FNAC with hormonal status and Her2 neu status and fit for treatment with surgery, cytotoxic chemotherapy, were considered eligible for the study. The total sample size 40 cases (stage 3 breast cancer) 20 patients in each group. If age $<40$ years USG b/l breast with axilla or histopathology size will be a guide to assess size of tumour ${ }^{12}$. If age $>40$ mammography or histopathology size will be a guide for tumour size ${ }^{12}$. The staging of the patient was done using TNM classification. ${ }^{13}$ All female patients of stage III carcinoma breast with histopathological negative margins (R0 resections) in their surgical interventions, and who gave consent to participate in study were included in the study.

\section{Exclusion Criteria}

- Patients who have already taken neoadjuvant therapy prior to admission.

- Patients with positive surgical margin.

- Patient not tolerating chemotherapy.

- $\quad$ Stage I breast cancer, Stage II breast cancer, Stage IIIc breast cancer, Stage IV breast cancer, patients with distant metastasis.

Patients in whom primary defect closure was possible were directly subjected to MRM followed by adjuvant chemotherapy. Patients in whom primary defect closure was not possible, they first underwent neoadjuvant first line of chemotherapy as advised by the tumour board discussion at AVBRH comprising of oncologist, onco-surgeon, pathologist, radiologist and radiotherapist. In case of response, patient underwent completion of chemotherapy neoadjuvantly. Patients who had partial response or clinical progressive disease to first line chemotherapy at the end of 3 cycles were subjected to $2^{\text {nd }}$ line of chemotherapy with paclitaxel. On completion of chemotherapy, Responders (clinical size decrement of more than $50 \%$ ) underwent modified radical mastectomy followed by adjuvant therapy. Nonresponders/partial responders/ progressive responders underwent palliative mastectomy followed by adjuvant chemotherapy patients were followed for 1 year once every 3 months. At every follow-up we examined local site, axilla, supraclavicular region, USG abdomen, and required investigations if symptoms of systemic metastasis were present.

\section{Statistical Analysis}

Statistical analysis was done by using descriptive and inferential statistics using chi square test, student's unpaired $\mathrm{t}$ test and Multiple Regression Analysis and software used in the analysis were SPSS 24.0 version and GraphPad Prism 7.0 version and $\mathrm{p}<0.05$ is considered as level of significance.

\section{RESULTS}

\section{Side, Age and Duration}

Patients in this study had carcinoma more of left breast $70 \%$ in adjuvant and $55 \%$ in neoadjuvant group) than compared to right breast. In the age wise distribution, highest number of patients in adjuvant group (30\% each) were in the range of 41-50 years and 61-70 years. Highest number of patients in neoadjuvant group (45\%) were in the range of $41-50$ years 
of age. In a total of 40 patients, the youngest patient in our study was 38 years old while the eldest was 68 years with the mean age of $52.73 \pm 11.25$ years in adjuvant group and in $50.20 \pm 7.55$ yrs. in neoadjuvant group. The median duration was 4 months in adjuvant group and 8 months in neoadjuvant group. Mean duration of lump was 7.35 months in adjuvant group and 11.95 months in neoadjuvant group. Both mean and median duration was higher in neo-adjuvant group as compared to adjuvant group. Neoadjuvant group had higher number of postmenopausal women $(85 \%)$ than adjuvant group (75\%).

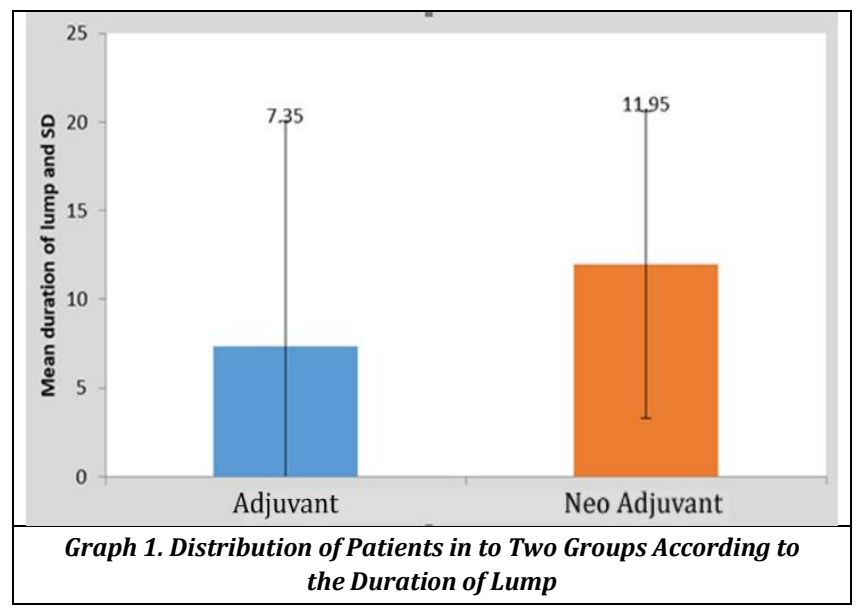

\section{T, N, and Staging in Each Group}

Out of 40 patients in both groups, maximum number of patients were in the tumour size group between $5.1-10 \mathrm{~cm}$. Most number of patients were clinically $\mathrm{N} 1$ at the time of presentation in both adjuvant ( $80 \%$ of patients) and in neoadjuvant ( $60 \%$ of patients) group. None of the patient was clinical N0 at the time of presentation in both the groups. On histopathology in adjuvant group, maximum number of patients (45\%) had 4-9 lymph nodes positive for metastasis. In neoadjuvant group also maximum number patients (30\%) had 4-9 lymph nodes positive for metastasis. In the adjuvant group $90 \%$ of patients belonged to stage IIIA while in neoadjuvant group only $50 \%$ patients belonged to stage IIIA. Rest patients in both group belonged to stage IIIB. This difference in adjuvant and neo-adjuvant group was statistically significant $(\mathrm{p}=0.005)$.

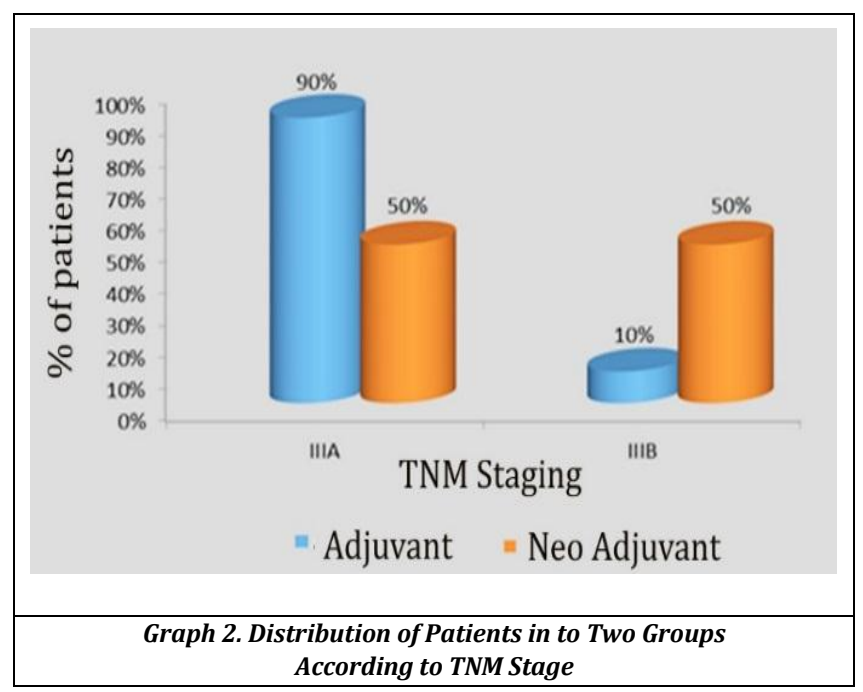

Lymphovascular Invasion, Type of Preferred Surgery, Chemotherapeutic Agent, Grade and Immunohistochemistry

Distribution of patients according to lymphovascular Invasion was done in which 18 out of 20 patients in adjuvant group and 14 out of 20 in neoadjuvant group were having Lymphovascular Invasion. This difference in number of patients in adjuvant and neoadjuvant group was statistically non-significant. However, when disease recurrence or disease metastasis/mortality was compared with lymphovascular invasion in neoadjuvant group, it was found to be statistically significant $(\mathrm{p}$ value $=0.022$ )

\begin{tabular}{|c|c|c|c|}
\hline Lymphovascular Invasion & Adjuvant & Neo-adjuvant & $\chi 2$-Value \\
\hline Yes & $18(90 \%)$ & $14(70 \%)$ & \multirow{3}{*}{$\begin{aligned} & 2.50 \\
\mathrm{p}= & 0.11, \mathrm{NS}\end{aligned}$} \\
\hline No & $2(10 \%)$ & $6(30 \%)$ & \\
\hline Total & $20(100 \%)$ & $20(100 \%)$ & \\
\hline
\end{tabular}

\begin{tabular}{|c|c|c|c|c|c|c|}
\hline & \multirow[t]{2}{*}{ Model } & \multicolumn{2}{|c|}{ 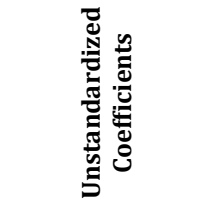 } & \multirow{2}{*}{ 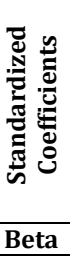 } & \multirow[t]{2}{*}{$\mathbf{t}$} & \multirow[t]{2}{*}{ 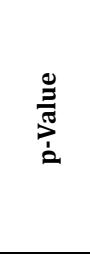 } \\
\hline & & B & Std. Error & & & \\
\hline \multirow{4}{*}{1} & $\begin{array}{c}\text { Mortality/ } \\
\text { Recurrence/Metastasis }\end{array}$ & -2.625 & 1.420 & & & \\
\hline & Type of malignancy & - & - & - & - & - \\
\hline & Lymphovascular invasion & 2.375 & 0.891 & 0.618 & 2.665 & $0.022, \mathrm{~S}$ \\
\hline & BR grading & 0.437 & 0.446 & 0.228 & 0.982 & $0.347, \mathrm{NS}$ \\
\hline \multicolumn{7}{|c|}{$\begin{array}{c}\text { Table 2. Multivariate Analysis of Mortality/Recurrence with Type of } \\
\text { Malignancy, Lymphovascular Invasion and BR Grading in } \\
\text { Neoadjuvant Group }\end{array}$} \\
\hline
\end{tabular}

Modified radical mastectomy was the most preferred surgery type. All 20 patients of adjuvant group and about 80 $\%$ of neoadjuvant group underwent modified radical mastectomy. Others underwent palliative mastectomy. In the adjuvant group, maximum number of patients received chemotherapy of CA + paclitaxel regimen (45\%). While in neoadjuvant group, maximum number of patients $(65 \%)$ received CAF regimen before surgery and paclitaxel (55\% patients) after surgery. Maximum number of patients in our participant population had grade II tumour on their histopathological report i.e. $80 \%$ in adjuvant group, while $50 \%$ patients in the neoadjuvant group.

In distribution of patients according to immunohistochemistry in our study population group maximum number of patients in both groups i.e. 55\% of adjuvant group and $45 \%$ of neoadjuvant group were of luminal type A and B. $20 \%$ patients in adjuvant group were of her2neu enriched type and $30 \%$ of patients were of her2neu enriched type in neo-adjuvant group. In both these groups least number of patients i.e. $25 \%$ patients were of triple negative type. 


\section{Outcome (Recurrence, Metastasis, Mortality)}

In comparison of outcome in both adjuvant and neo-adjuvant chemotherapy, $5 \%$ patients of adjuvant group developed metastasis and died succumbing to it while another $5 \%$ developed recurrence during follow up. In the neoadjuvant group 35\% patients developed distant metastasis or died due to disease while another $5 \%$ patient developed local recurrence in axilla for the disease. This difference in the outcome of two groups was statistically significant with $\mathrm{p}$ value of 0.013 . The mean follow-up in neoadjuvant was 5.3 months whereas mean follow up of adjuvant group was 10.65 months. There is statistical significance in follow-up between neo-adjuvant and adjuvant chemotherapy.

\begin{tabular}{|c|c|c|c|}
\hline Outcome & Adjuvant & Neoadjuvant & $\mathbf{p}$ \\
\hline Mortality due to disease & $0(0 \%)$ & $3(15 \%)$ & \multirow{5}{*}{$\begin{array}{c}6.14 \\
p=0.013\end{array}$} \\
\hline Distant Metastasis but alive & $1(5 \%)$ & $4(20 \%)$ & \\
\hline Locoregional recurrence & $1(5 \%)$ & $1(5 \%)$ & \\
\hline Lost to follow up & $0(0 \%)$ & $1(5 \%)$ & \\
\hline Total & $2(10 \%)$ & $9(45 \%)$ & \\
\hline \multicolumn{4}{|c|}{$\begin{array}{c}\text { Table 3. Distribution of Patients in the Two Groups According to } \\
\text { Mortality/Metastasis/Recurrence }\end{array}$} \\
\hline
\end{tabular}

\begin{tabular}{|c|c|c|c|}
\hline $\begin{array}{c}\text { Stage IIIA Patients in Adjuvant } \\
\text { vs Neoadjuvant }\end{array}$ & $\begin{array}{c}\text { Adjuvant } \\
\text { Group (n=20) }\end{array}$ & $\begin{array}{c}\text { Neoadjuvant } \\
\text { Group (n=20) }\end{array}$ & p \\
\hline Total no of patients of stage IIIA in & $18(90 \%)$ & $10(50 \%)$ & $\mathbf{0 . 0 2 2}$ S \\
\hline $\begin{array}{c}\text { Metastasis/mortality/recurrence in } \\
\text { stage IIIA patients }\end{array}$ & $2(10 \%)$ & $3(20 \%)$ & \\
\hline \multicolumn{3}{|c|}{ Table 4. Distribution of Patients of Only Stage IIIA } \\
in Both the Groups with Their Outcome \\
\hline
\end{tabular}

\section{DISCUSSION}

In our study, BR grading, luminal status or triple negative status, laterality of disease, age at presentation, histopathological type of malignancy were a nonsignificant contributing factor in determining prognosis of disease.

\section{Lymphovascular Invasion}

In our study, $90 \%$ patients in adjuvant group had lymphovascular invasion whereas only $70 \%$ patients in neoadjuvant group had lymphovascular invasion. This above data is though statistically nonsignificant. In the adjuvant group out of 18 patients who had lymphovascular invasion present, 2 patients developed either metastasis or recurrence. In the neo-adjuvant group out of 14 patients who had LVI, 7 patients developed recurrence/metastasis/death during treatment or follow up, while 1 patient was lost to follow up in neoadjuvant. In a multivariate analysis between metastasis/local recurrence/mortality vs type of malignancy/LVI/BR grading, the correlation between metastasis/local recurrence/ mortality was found to be significant with LVI in neoadjuvant group ( $p$ value 0.022).Thus presence of lymphovascular invasion is a sign of poor prognosis. However, the relation was non-significant in the adjuvant group. In the study by Ryu et al,14 out of 187 patients, $35 \%$ patients showed LVI. The LVI group tended to have advanced status in terms of disease burden (clinical stage III) and had statistical significance. In the univariate analysis of association with recurrence (locoregional/distant) LVI ( $p$ value <0.001) showed statistically significant differences. Similar results were found with statistically significant differences in the study by Liu et al, $^{15}$ where in the univariate analysis, presence of LVI was significantly associated with worse progression free survival ( $p$ value $<0.01)$.

\section{Outcome (Mortality/Metastasis/Recurrence)}

In our study, 3 patients (15\%) of neoadjuvant group died due to disease. Metastasis was found in $1(5 \%)$ patient in adjuvant group while $4(20 \%)$ patient in neoadjuvant group. Recurrence was found in $1(5 \%)$ case each in adjuvant and neo-adjuvant group. The death in neoadjuvant group was due to distant metastasis. In the neoadjuvant group, 3 patients developed bony metastasis first, while 2 patients developed liver metastasis first. One patient developed lung metastasis. One patient developed seizures and brain metastasis. 1 patient in neoadjuvant group was lost to follow-up. The comparison in between the two groups of adjuvant and neoadjuvant in terms of poor prognosis (mortality/ metastasis/recurrence) was found to be statistically significant ( $p$ value of 0.013 ) (table 3 ). Hence our study shows that overall outcome in terms of disease recurrence (distant/local) is poor when neoadjuvant chemotherapy is given as compared to adjuvant therapy in stage IIIA \&IIIB carcinoma breast.

Separately, when we compared outcome (mortality/ metastasis/recurrence) in only stage IIIA patients of both the groups(table 4), the recurrence and metastasis happened in two patients in adjuvant group who belonged to stage IIIA $(2 / 18,11 \%)$. While in neoadjuvant group 3 out of 10 patients developed mortality/metastasis/recurrence. This comparison of outcome among stage III A patients among adjuvant and neoadjuvant group was found to be statistically significant ( $p$ value 0.022 ) which means that adjuvant chemotherapy plan is better for stage IIIA patients than neoadjuvant chemotherapy plan.

In Deo et al study, ${ }^{16}$ metastasis was found in $26 \%$ patients receiving neoadjuvant therapy and in $13.8 \%$ patients receiving adjuvant therapy. Local recurrence was seen in 2 patients (4\%) in neoadjuvant group and 4 patients (8\%) in adjuvant group. Mortality due to disease was seen in 11 (22\%) patients in neoadjuvant group and $9(18 \%)$ patients in adjuvant group. This study also shows that there is higher rate of disease recurrence (distant and local) and mortality in neoadjuvant arm as compared to adjuvant arm. Meta-analysis done by Davide mauri et $\mathrm{al}^{17}$ on comparison between adjuvant versus neoadjuvant chemotherapy in breast cancer found no statistically or clinically significant difference between neoadjuvant and adjuvant therapy arms associated with death, disease progression or distant metastasis. However neoadjuvant chemotherapy was statistically significantly associated with an increased risk of locoregional disease recurrences compared with adjuvant group especially in studies where more patients in neoadjuvant group than adjuvant group received radiation therapy without surgery.

In the study of adjuvant chemotherapy only, Casper et al ${ }^{18}$ found 7 of 41 patients (17\%) had local recurrence of which 5 patients later developed distant metastasis. Saarto et $\mathrm{al}^{19}$ in their study of adjuvant regimens in carcinoma breast over a 
follow-up of 8 years found that distant metastasis occurred in $48 \%$ of patients while $23 \%$ had local recurrence. All local recurrence patients later developed distant metastasis. Marrow et $\mathrm{al}^{20}$ in their study on effectivity of neoadjuvant chemotherapy found that out of 31 patients, $3(10 \%)$ patient developed metastasis who completed therapy and 4 patients $(13 \%)$ of them had local recurrence. While 2 patients did not complete their complete course of treatment. There are several factors contributing to poor prognosis (disease recurrence/mortality) are triple negative status, LVI presence, her2 neu overexpression. Of these, in our study LVI was statistically significantly associated with poor outcome in neoadjuvant group in terms of distant metastasis and locoregional relapse. Other parameters were statistically nonsignificant in our study. This could be because of low sample size.

\section{Limitations}

1. Small sample size.

2. Shorter duration of follow-up done in this study.

3. Study is conducted in the rural setup hospital, where patients are relatively less educated and economically from weaker section. Hence the compliance to the treatment is poor either due to lack of education or due to monetary issues in reporting to healthcare setups. Also, this leads to late presentation at hospital and often defaults of cycles and loss to follow-up.

4. Adjuvant group had mostly stage IIIA and neoadjuvant group had more of higher staged patients, mainly of stage IIIB. Thus, the poor outcome of neoadjuvant group could be due this bias. This inequality in stage of each group occurred because large operable breast cancers mostly belong to stage IIIA and hence they underwent adjuvant chemotherapy while, non-operable patients fall under IIIB who had to go neoadjuvant chemotherapy first to downgrade and make the lump operable.

5. Three patients in adjuvant group and five patients in neoadjuvant group are still under follow up and their 1 year of follow up set by the protocol of this study is still ongoing. Hence comment on their outcome will be too early to come to a conclusion.

\section{CONCLUSIONS}

Adjuvant chemotherapy is better in outcome in terms of locoregional recurrence and distant metastasis for stage IIIA \& IIIB carcinoma breast than neoadjuvant chemotherapy, provided surgery with primary skin closure is feasible. Lymphovascular invasion is a marker for poor prognosis in carcinoma breast.

\section{REFERENCES}

[1] Ferlay J, Soerjomataram I, Dikshit R, et al. Cancer incidence and mortality worldwide: sources, methods and major patterns in GLOBOCAN 2012. Int J Cancer 2015;136(5):E359-E86.
[2] Porter PL. Global trends in breast cancer incidence and mortality. Salud Publica Mex 2009;51 Suppl 2:S141-6.

[3] Babu GR, Lakshmi SB, Thiyagarajan JA. Epidemiological correlates of breast cancer in South India. Asian Pac J Cancer Prev 2013;14(9):5077-83.

[4] Ali I., Wani WA, Saleem K. Cancer scenario in India with future perspectives. Research article. Cancer Therapy 2011;8:56-70.

[5] Reddy KS, Shah B, Varghese C, et al. Responding to the threat of chronic diseases in India. Lancet 2005;366(9498):1744-9.

[6] Bagri-Manjrekar K, Chaudhary M, Sridharan G, et al. In vivo autofluorescence of oral squamous cell carcinoma correlated to cell proliferation rate. Journal of Cancer Research and Therapeutics 2018;14(3):553-8.

[7] Agarwal A, Bhola N, Kambala R, et al. Touch imprint cytology: can it serve as an alternative to frozen section in intraoperative assessment of cervical metastasis in oral squamous cell carcinoma? Journal of Oral and Maxillofacial Surgery 2019;77(5):994-9.

[8] Thakur NA, Humne AY, Godale LB. Delay in presentation to the hospital and factors affecting it in breast cancer patients attending tertiary care center in Central India. Indian Journal of Cancer 2015;52(1):102-5.

[9] Lamture YR, Balaji S, Md Shahabuddin. Carcinoma of breast-a study profile. Journal of Evolution of Medical and Dental Sciences (JEMDS) 2018;7(45):4857-61.

[10] National Comprehensive Cancer Network. Version 3. 2019 - September 6, 2019. https://www2.trikobe.org/nccn/guideline/breast/english/breast.pdf

[11] Breast_Cancer.pdf.

[12] https://acsjournals.onlinelibrary.wiley.com/doi/10.100 2/cncr.28012

[13] Breast Cancer Staging: TNM classification for breast cancer. Author: Sparano JA. Chief Editor: Lee MC. Updated: Jun 08, 2019.

[14] Ryu YJ, Kang SJ, Cho JS, et al. Lymphovascular invasion can be better than pathologic complete response to predict prognosis in breast cancer treated with neoadjuvant chemotherapy. Medicine (Baltimore) 2018;97(30):e11647.

[15] Liu YL, Saraf A, Lee SM, et al. Lymphovascular invasion is an independent predictor of survival in breast cancer after neoadjuvant chemotherapy. Breast Cancer Res Treat 2016;157(3):555-64.

[16] Deo SVS, Bhutani M, Shukla NK, et al. Randomized trial comparing neo-adjuvant versus adjuvant chemotherapy in operable locally advanced breast cancer (T4b N0-2 M0). Journal of Surgical Oncology 2003;84(4):192-7.

[17] Mauri D, Pavlidis N, Ioannidis JPA. Neoadjuvant versus adjuvant systemic treatment in breast cancer: a metaanalysis. J Natl Cancer Inst 2005;97(3):188-94.

[18] Casper ES, Guidera CA, Bosl GJ, et al. Combined modality treatment of locally advanced breast cancer: adjuvant combination chemotherapy with and without doxorubicin. Breast Cancer Res Treat 1987;9(1):39-44. 
[19] Saarto T, Blomqvist C, Tiusanen K, et al. The prognosis of stage III breast cancer treated with postoperative radiotherapy and adriamycin-based chemotherapy with and without tamoxifen: Eight year follow-up results of a randomized trial. European Journal of Surgical Oncology 1995;21(2):146-50.
[20] Morrow M, Braverman A, Thelmo W, et al. Multimodal therapy for locally advanced breast cancer. Arch Surg 1986;121(11):1291-6. 